\title{
Minidam-A Miniature to Isolation
}

\author{
Swathika M C L *, Jayanthi Mungara**, Akila $V^{* * * *}$, Dharini $V^{* * * * *}$ \\ *Post graduate student, Ragas Dental College \\ ** Head of the Department, Ragas Dental College \\ *** Senior Lecturer, Thai Moogambigai Dental College \\ ***** Post graduate student, Ragas Dental College \\ DOI: 10.29322/IJSRP.11.05.2021.p11325 \\ http://dx.doi.org/10.29322/IJSRP.11.05.2021.p11325
}

\begin{abstract}
INTRODUCTION: The complexity of oral environment presents many obstacles in performing dental treatment procedures. To minimize them, proper isolation is required to control operating field as well as provide safe and quality treatment. Rubber dam isolation has been a gold standard for several years. However, general dentists and specialists may not use rubber dam owing to the difficulty in placement and cost.
\end{abstract}

AIM: The aim of this study is to analyse the acceptance of minidam using subjective and objective anxiety scales respectively.

MATERIALS AND METHODS: The study included 30 children between the age group of 6-8 years who had come to our Department requiring restoration for the carious lesion in upper and lower primary molars. Anxiety and acceptance of the new isolation method was assessed subjectively \& objectively by using Facial Image Scale (FIS) \& FLACC Behavioural assessment scale respectively. In addition, outcome like time required for the placement of minidam was recorded.

RESULTS: The results showed that $p=0.00$, less than 0.05 there is a statistically significant difference between Facial Image Scale before \& after treatment., thereby reveals that the children were comfort with the placement of minidam. Also, there is a strong correlation between subjective and objective score.

CONCLUSION: Results showed that minidam could offer effective isolation with limited treatment procedure and seems to be comfort for the children.

Index Terms-Minidam, Anxiety, Carious lesions, Primary molar

\section{INTRODUCTION}

he oral environment needs to be adequately controlled to prevent it from interfering with the execution of any dental procedure. This control is attained through isolation.

Traditionally, tooth isolation from oral fluids (saliva, blood, gingival fluid etc.) during restorative procedures has been accepted as an important step for clinical excellence in dentistry1. Complete isolation during the procedure is critical for longevity of the restoration and therefore this material is the choice where isolation can be achieved2.

Rubber dam isolation has been a gold standard for several years as it offers various advantages including antisepsis, moisture control, retraction and protection of soft tissues, protection against aspiration of endodontic instruments or toxic materials, prevent cross infection and protect the operator from legal responsibility, if accidents occur. However, it is seen that dentists do not use rubber dam routinely, more so in children4. The reasons for refraining from the use of rubber dam are multifold. Sanghvi., et al. reported factors such as time consuming, patient compliance, inexperience with rubber dam usage and cost as reasons for dentists not using the rubber dam frequently5.

Newer isolation techniques and materials are being explored that would reduce the difficulty to the operator and the patient and at the same time offer satisfactory isolation. Minidam (DMG America LLC) was introduced recently to aid in isolation in restoration of carious lesions. It is a two-tooth slot system, latex free and is stabilized without the use of clamps.

Consequently, the aim of this study is to analyse the acceptance of minidam using subjective and objective anxiety scales respectively

\section{IDENTIFY, RESEARCH AND COLLECT IDEA}

Methods:

The study comprises of 30 children who attended outpatient clinic of the Department of Pedodontics \& Preventive dentistry. Children between the age of 6-8 yrs; having good systemic health and with atleast one carious lesion in upper \& lower primary molars were included in the study. Participants who had other dental problems like pulpitis \& its sequlae, Medically compromised

children and children with Frankl's rating negative and definitely negative were excluded from the study. Parents were explained regarding the purpose and scope of the research and informed consents were obtained if they were willing to allow their child's participation in the study.

Operating field made isolated with the application of minidam, The Minidam was stretched over the tooth to be treated and dental floss was placed interdentally to allow proper seating of the material. Meanwhile, anxiety level was assessed by using Facial Image Scale ( FIS); comprises a row of five faces ranging from very happy to very sad, in which children was made to point 
out at which face they felt like most at the moment before and after the treatment. Child's behaviour was evaluated by using FLACC Scale ( Face,Leg,Activity,Cry, Consolability), each category is scored on the $0-2$ scale, which results in a total score of 0-10.The lesser the score, the better was the child's behaviour which suggestive of the comfortability to the child on the

\section{WRITE DOWN YOUR STUDIES AND FINDINGS}

On statistical analysis, result reveals that $\mathrm{p}=0.00$, less than 0.05 there is a statistically significant difference between Facial Image Scale before \& after treatment, suggesting that the patient was happy and comfort with the placement of minidam. Time duration for the placement was minimal, mean duration was 5mins. When checked for co-relation, it also showed that there is a strong correlation between subjective and objective score.

\section{Discussion:}

The oral cavity is a complex environment which is surrounded on all sides by hard and soft tissues. Most of the dental materials used are hydrophobic in nature. Hence for the success of any dental treatment a thorough isolated field is mandatory. Here, the use of minidam provides good isolation by seepage of saliva during the restorative treatment.

In our study, the time taken for the placement of minidam was minimal and skill needs to be acquired with practice. The primary finding from our study was that children exhibited good behaviour and less perceived pain on the placement of minidam. On the other hand, Improper fitting of rubber dam clamps can cause impingement of gingiva, discomfort \& leakage of saliva which can lead to poor cooperation of children. 6 Similar findings was reported by Amman et al 7

Therefore, clamp free isolating technique like minidam would reduce the discomfort considerably, this was in accordance with the study done by Sehgal \& Uma8.

Even though the results of the study showed that minidam among children have achieved higher rates of acceptance, it also has its considerations:

1. Minidam limits only to few teeth for isolation purpose.

2. Cannot withstand much of spaced dentition

3 . Loss of adjacent teeth is contraindicated.

Our findings should be viewed through certain limitations of the study which comprises of 30 children with the age group of 6-8 yrs. Strength of the study was its uniqueness, as we could not find any published literature on Minidam. Pediatric dentists are always on the lookout for an adequately effective isolating technique that will reduce the patient discomfort and anxiety. And such study will offer better insight into the use of this material.

\section{CONCLUSION}

placement of minidam during the restorative procedure. Time required for the placement of isolating material was recorded in minutes by the trained assistant with the help of an electronic stop watch.

Even though the study shows limitations, it was unanimously accepted by most of the children. Anyhow, further investigations has to be performed in order to acquire not only the acceptance but also to appreciate the clinical result.

\section{REFERENCES}

[1] Cajazeria MR, Sabola TM \& Cople Maia. Influence of the operatory field isolation technique on tooth-colored direct dental restorations. American Journal of Dentistry.2014:27;3

[2] Antony K., et al. "Longevity of dental amalgam in comparison to composite materials". GMS Health Technology Assessment 13.4 (2008): 1-12.

[3] Priyanka R Sehgal \& Uma B Dixit. Use of Minidam for Isolation in Restoration of proximal Carious lesion. Acta Scientific Dental Sciences 3.5(2019): 105-109.

[4] Roshan D., et al. "Changes in dentists' attitudes and practice in paediatric dentistry". European Journal of Pediatric Dentistry 4.1 (2003): 21-27.

[5] Sanghvi AM., et al. "A cross-sectional study on frequency of rubber dam usage among dentists practicing in Maharashtra,India". Saudi Endodontic Journal 8.1 (2018): 39-43.

[6] Innes N. "Rubber dam use less stressful for children and dentists".Evidence Based Dentistry 13.2 (2012): 48.

[7] Amman P., et al. "Influence of rubber dam on objective and subjective parameters of stress during dental treatment of children and adolescents - a randomized controlled clinical pilot study". International Journal of Paediatric Dentistry 23.2(2012): 110-115.

[8] Priyanka R Sehgal and Uma B Dixit. "Use of Minidam for Isolation in Restoration of Proximal Carious Lesion". Acta Scientific Dental Sciences 3.5(2019): 105-109.

\section{AUTHORS}

First Author - Dr. Swathika.M.C.L, Post Graduate Student, Ragas Dental college and Hospital, Chennai.

ashwinilatharasu@gmail.com

Second Author - Dr. Jayanthi Mungara,MDS,Head of the Department, Ragas Dental college and Hospital, Chennai and email address.

Third Author - Dr. Akila, MDS, Senior Lecturer, Thai Moogambigai Dental college and hospital .

akilaveerapandian@gmail.com

Fourth Author - Dr. Dharini,Post Graduate Student, Ragas

Dental college and Hospital, Chennai .

dharinivenkat1996@gmail.com

Correspondence Author - Dr. Swathika.M.C.L,Post Graduate Student, Ragas Dental college and Hospital, Chennai. ashwinilatharasu@gmail.com. 9962086006 